\title{
Correction: Mitochondrial Morphology and Fundamental Parameters of the Mitochondrial Respiratory Chain Are Altered in Caenorhabditis elegans Strains Deficient in Mitochondrial Dynamics and Homeostasis Processes
} Anthony L. Luz, John P. Rooney, Laura L. Kubik, Claudia P. Gonzalez,
Dong Hoon Song, Joel N. Meyer

In Figs 2, 3, 4 and 5, and S1, S4, S5, S7, S9, S10, S11, S12, S13, S14, S16, S17, S18, S19 and S20 Figs, the $\mathrm{y}$-axes are incorrectly labeled " $\mathrm{pmol} / \mathrm{min} / \mathrm{mg}$ protein", when they should be labeled "nmol/min/mg protein". Please see the corrected files here.

\section{G openaccess}

Citation: Luz AL, Rooney JP, Kubik LL, Gonzalez CP, Song DH, Meyer JN (2016) Correction: Mitochondrial Morphology and Fundamental Parameters of the Mitochondrial Respiratory Chain Are Altered in Caenorhabditis elegans Strains Deficient in Mitochondrial Dynamics and Homeostasis Processes. PLoS ONE 11(12): e0168738. doi:10.1371/journal.pone.0168738

Published: December 15, 2016

Copyright: ๑ 2016 Luz et al. This is an open access article distributed under the terms of the Creative Commons Attribution License, which permits unrestricted use, distribution, and reproduction in any medium, provided the original author and source are credited. 

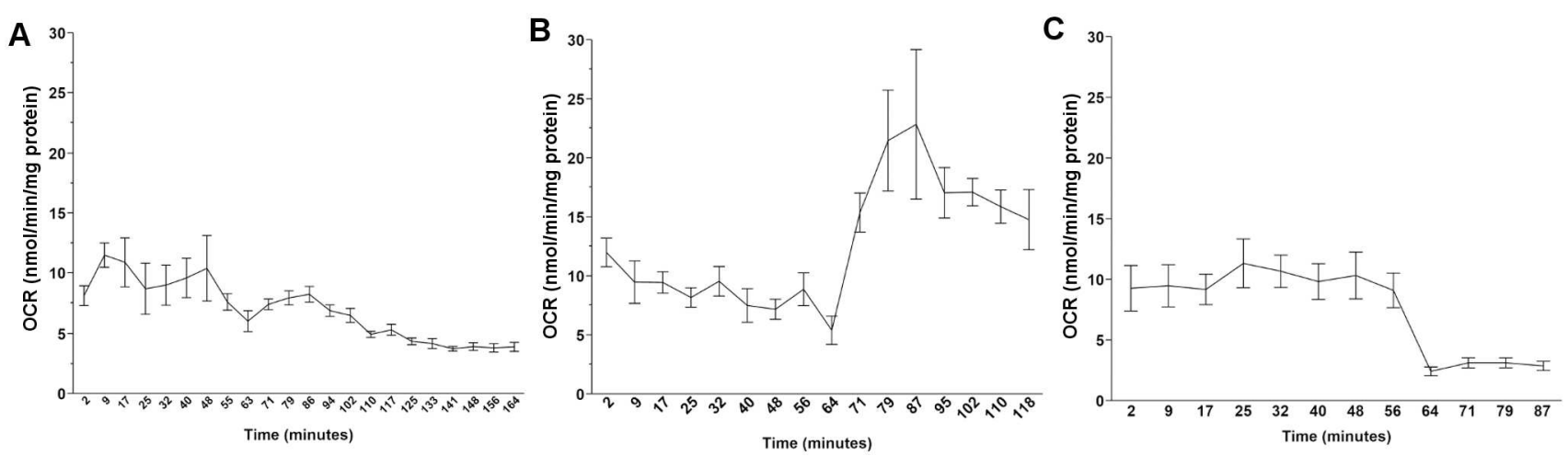

Fig 2. Representative Seahorse XFe24 output data for L4 N2 nematodes dosed with (A) DCCD, (B) FCCP, and (C) sodium azide. doi:10.1371/journal.pone.0168738.g001

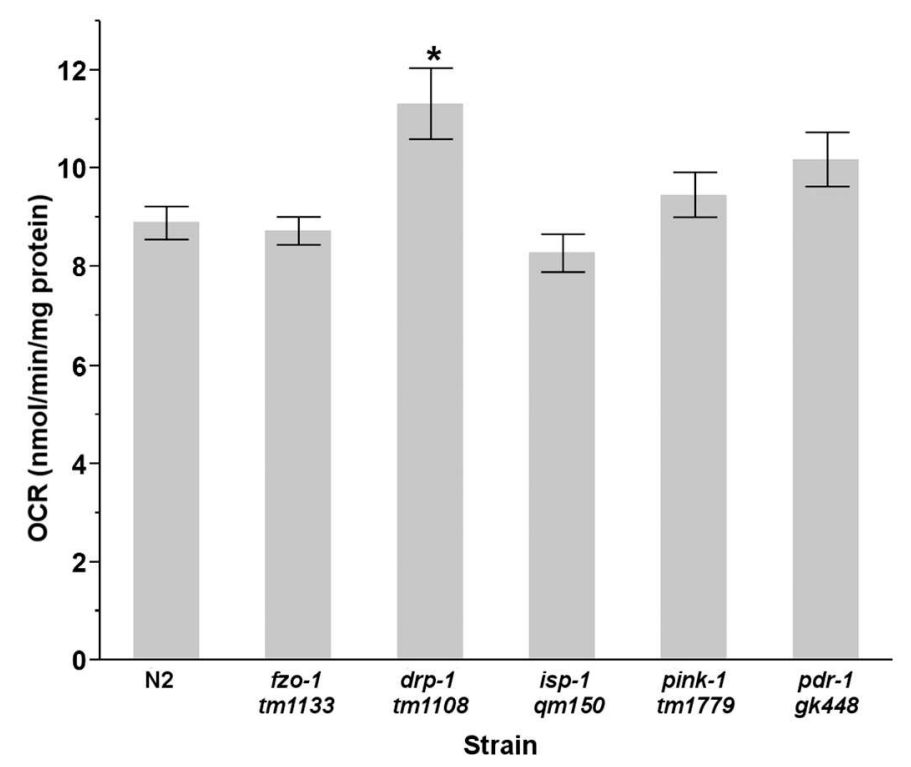

Fig 3. Basal OCR is elevated in L4 drp-1 nematodes. Statistical significance was analyzed via a one way ANOVA (main effect of strain, $P<0.0001)(n=31-45)$. Asterisks $(*)$ denote statistical significance. Bars \pm SEM.

doi:10.1371/journal.pone.0168738.g002 

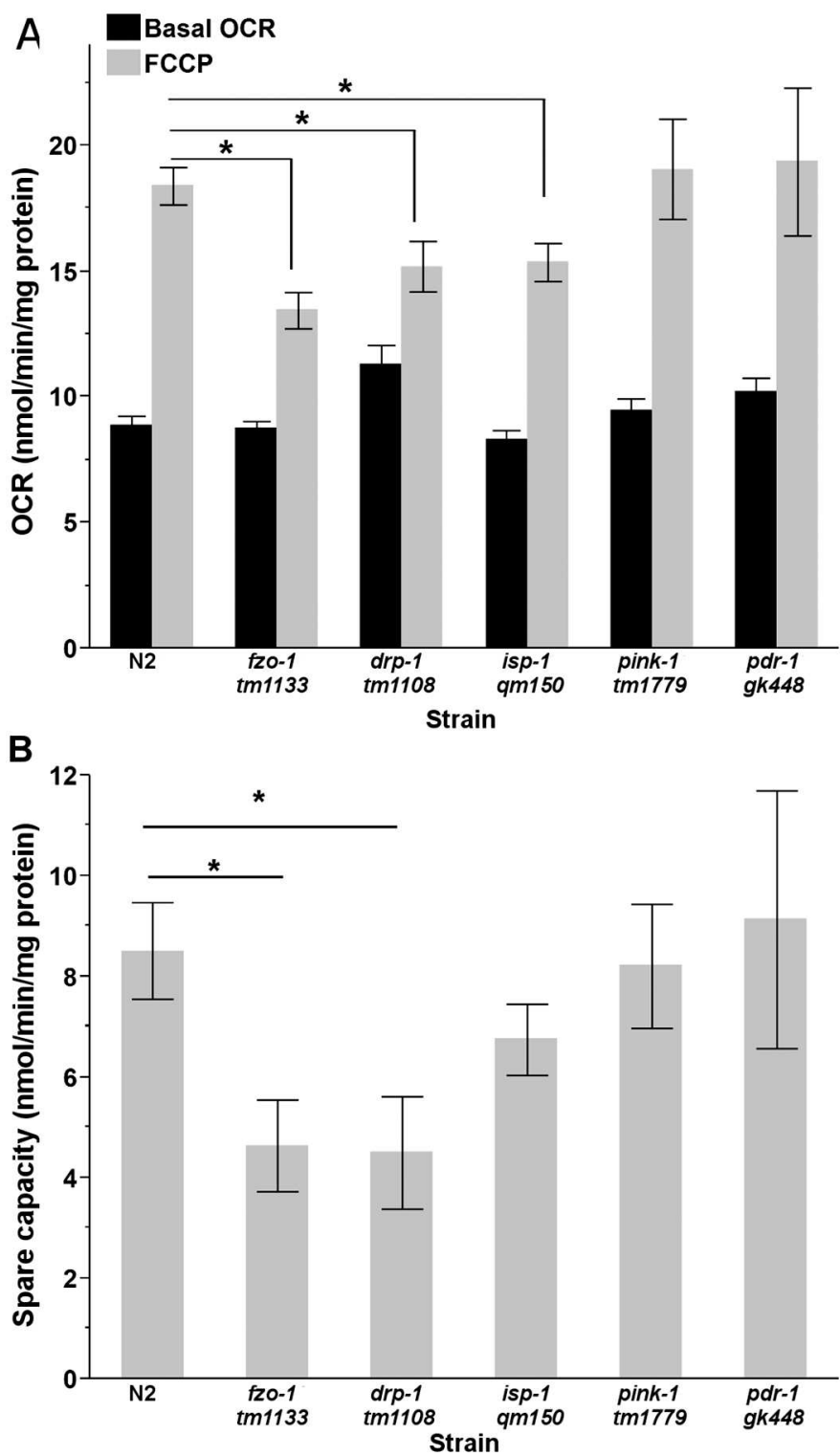

Fig 4. Maximal and spare respiratory capacity in L4 nematodes. (A) Treatment with FCCP caused a significant increase in OCR in all strains (two way ANOVA, main effects of strain, treatment and their interaction, $\mathrm{P}<0.0001$ for all); however, L4 fzo-1, drp-1 and isp-1 had reduced maximal respiratory capacity compared to N2 nematodes (Student's t-test, $p=0.03, p<0.0001, p=0.01$, respectively). (B) Spare respiratory capacity was reduced in $f z o-1$ and $d r p-1$, compared to wild-type nematodes (one way ANOVA, $P=0.022) .(n=12-20)$. Asterisks $\left({ }^{*}\right)$ denote statistical significance. Bars \pm SEM.

doi:10.1371/journal.pone.0168738.g003 

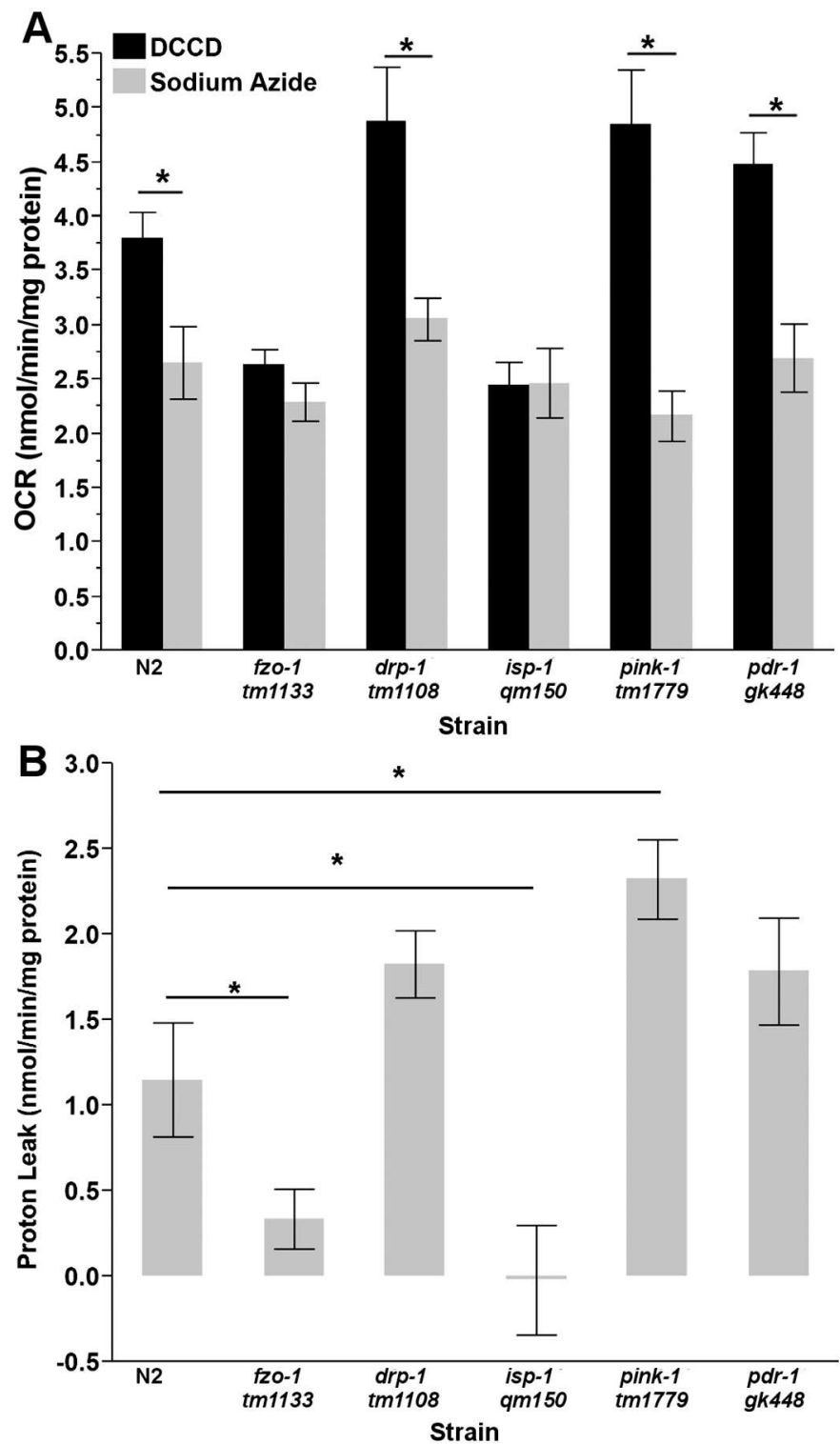

Fig 5. Proton leak in L4 nematodes. (A) Sodium azide and DCCD caused significantly different reductions in OCR in N2, drp-1, pink-1, and pdr-1 nematodes (two way ANOVA, main effects of strain $(\mathrm{P}<0.0001)$, treatment $(P<0.0001)$ and their interaction $(p=0.0002))$, while fzo-1 and isp-1 responses were not significantly different. (B) $L 4 f z o-1$ and isp-1 nematodes have reduce proton leak, while pink-1 C. elegans have increased leak (one way ANOVA, $P<0.0001)$. $(n=8-12)$. Asterisks $\left(^{*}\right)$ denote statistical significance. Bars \pm SEM.

doi:10.1371/journal.pone.0168738.g004

\section{Supporting Information}

S1 Fig. Response of L4 N2 nematodes to sodium azide alone and post-FCCP. Response to sodium azide was assessed statistically with a one way ANOVA $(\mathrm{P}=0.0005)$. Asterisks $\left(^{*}\right)$ denote statistical significance. Bars \pm SEM.

(TIFF) 
S4 Fig. Titration of Dicyclohexylcarbodiimide in L4 N2 nematodes. Significance assessed with a one way ANOVA $(\mathrm{P}<0.0001)$, followed by student's T-tests for pairwise comparisons. Asterisks $\left({ }^{*}\right)$ denote statistical significance. Bars \pm SEM.

(TIFF)

S5 Fig. Effect of DMSO concentration of efficacy of $20 \mu \mathrm{M}$ DCCD. Significance assessed with a one way ANOVA $(\mathrm{P}<0.0001)$, followed by student's T-tests for pairwise comparisons. Asterisks $\left(^{*}\right)$ denote statistical significance. Bars \pm SEM.

(TIFF)

S7 Fig. Titration of FCCP in L4 N2 nematodes. Significance assessed with a one way ANOVA (main effect of treatment, $\mathrm{P}<0.0001$ ). Asterisks $\left({ }^{*}\right)$ denote statistical significance. Bars \pm SEM.

(TIFF)

S9 Fig. Titration of sodium azide in L4 N2 nematodes. Significance assessed with a one way ANOVA (main effect of treatment, $\mathrm{P}<0.0001$ ). Asterisks $\left({ }^{*}\right)$ denote statistical significance. Bars \pm SEM.

(TIFF)

S10 Fig. Basal OCR is elevated in drp-1 and reduced in isp-1 L4 C. elegans on a per nematode basis. Asterisks $\left(^{*}\right)$ denote statistical significance. Bars \pm SEM.

(TIFF)

S11 Fig. ATP coupled respiration. (A) $20 \mu \mathrm{M}$ DCCD caused a significant reduction in OCR in all strains (two way ANOVA, main effects of strain and treatment, $\mathrm{P}<0.0001$ for both, but not their interaction). (B) A trend in increased ATP coupled respiration was observed in fzo-1 nematodes (one way ANOVA, $\mathrm{P}=0.07)$. $(\mathrm{n}=12-16)$. Asterisks $\left(^{*}\right)$ denote statistical significance. Bars \pm SEM.

(TIFF)

S12 Fig. ATP coupled respiration per nematode. (A) $20 \mu \mathrm{M}$ DCCD caused a significant reduction in OCR in all strains (two way ANOVA, main effects of strain and treatment, $\mathrm{P}<0.0001$ for both, but not their interaction), (B) but no significant effect on ATP-linked respiration was observed. Asterisks $\left({ }^{*}\right)$ denote statistical significance. Bars \pm SEM.

S13 Fig. Maximal and spare respiratory capacity in L4 C. elegans on a per nematode basis. (A) $f z o-1$, isp-1, and $d r p-1$ nematodes have a significantly reduced FCCP response (two way ANOVA, main effects of strain, treatment, and their interaction, $\mathrm{P}=0.0001$ for all) $(\mathrm{A})$ and (B) spare respiratory capacity. Asterisks $\left({ }^{*}\right)$ denote statistical significance. Bars \pm SEM. (TIFF)

S14 Fig. Proton leak per L4 nematode. (A) Effect of sodium azide and DCCD on OCR (two way ANOVA, $\mathrm{P}>0.05$ ) and (B) proton leak per nematode measured (two way ANOVA, $\mathrm{P}>0.05)$.

S16 Fig. Proton leak per L4 nematode. DMSO had no effect on OCR in any of the strains tested (two way ANOVA, P>0.05). Bars \pm SEM.

(TIFF) 
S17 Fig. Basal OCR and sodium azide response. Sodium azide caused a significant reduction in OCR in all strains tested (one was ANOVA, $\mathrm{P}<0.0001$ ). Bars \pm SEM.

(TIFF)

S18 Fig. Oligomycin pre-incubation with bus-8-deficient nematodes. A 12 hour preincubation with oligomycin caused a significant reduction in OCR (one way ANOVA, $\mathrm{P}<0.0001)$. Bars \pm SEM.

S19 Fig. Oligomycin titration with bus-8-deficient nematodes. Treatment with oligomycin caused a significant reduction in OCR (one way ANOVA, $\mathrm{P}=0.0007$ ). Bars \pm SEM. (TIFF)

S20 Fig. DCCD titration with bus-8-deficient nematodes. Treatment with DCCD caused a significant reduction in OCR (one way ANOVA, $\mathrm{P}<0.0001$ ). Bars \pm SEM.

(TIFF)

\section{Reference}

1. Luz AL, Rooney JP, Kubik LL, Gonzalez CP, Song DH, Meyer JN (2015) Mitochondrial Morphology and Fundamental Parameters of the Mitochondrial Respiratory Chain Are Altered in Caenorhabditis elegans Strains Deficient in Mitochondrial Dynamics and Homeostasis Processes. PLoS ONE 10(6): e0130940. doi: 10.1371/journal.pone.0130940 PMID: 26106885 